\title{
ESTIMATES FOR THE NORMS OF SOLUTIONS OF DIFFERENCE SYSTEMS WITH SEVERAL DELAYS
}

\author{
RIGOBERTO MEDINA
}

Received 1 August 2003

\begin{abstract}
We derive explicit stability conditions for time-dependent difference equations with several delays in $C^{n}$ (the set of $n$ complex vectors) and estimates for the size of the solutions. The growth rates obtained here are not necessarily decay rates.
\end{abstract}

2000 Mathematics Subject Classification: 39A10.

1. Introduction. Stability of systems of difference equations with delays has been discussed by many authors, for example, see Gil' and Cheng [6], Zhang [11], Elaydi and Zhang [5], Pituk [10], Agarwal [1], and the references therein.

In the stability literature, one can find two major trends; stability using the first approximation Lyapunov method and the direct Lyapunov functional method. For this latter trend, see Zhang and Chen [12], Crisci et al. [4], Lakshmikantham and Trigiante [7], and Agarwal and Wong [2]. By this method many very strong results are obtained. But finding Lyapunov functionals is usually a difficult task.

In this note, we consider a class of perturbed difference equations with several delays and, by means of a Gronwall inequality and the recent estimates for the powers $A^{k}$ of a constant matrix $A$ established in Corduneanu [3], we derive explicit stability conditions. Further, we suppose that the unperturbed linear difference equations have a bounded growth. Actually, this work is an extension of Medina [8] to time-dependent difference equations with several delays.

2. Preliminary facts. Let $C^{n}$ be the set of $n$ complex vectors endowed with a norm $\|\cdot\|$. Let $A_{k}(k=1,2, \ldots)$ be $n \times n$-complex matrices.

Consider in $C^{n}$ the equation

$$
u_{j+1}=A_{j} u_{j}+f_{j}\left(u_{j-\sigma_{1}}, \ldots, u_{j-\sigma_{p}}\right), \quad j=0,1, \ldots,
$$

where $p \geq 1, \sigma_{1}, \sigma_{2}, \ldots, \sigma_{p}$ are nonnegative integers such that $0=\sigma_{1}<\sigma_{2}<\cdots<$ $\sigma_{p}, \sigma_{i} \in Z^{+}$, and $Z^{+}$is the set of nonnegative integers, $f_{j}, j=0,1,2, \ldots$, maps $C^{n p}$ into $C^{n}$.

We will consider (2.1) subject to the initial conditions

$$
u_{j}=\tau_{j}, \quad j=-\sigma_{p},-\sigma_{p}+1, \ldots, 0 .
$$


It is assumed that there are nonnegative sequences $q_{l}(l=1,2,3, \ldots, p)$ such that

$$
\left\|f_{j}\left(u_{j-\sigma_{1}}, \ldots, u_{j-\sigma_{p}}\right)\right\| \leq \sum_{l=1}^{p} q_{l}(j)\left\|u_{j-\sigma_{l}}\right\|^{m}, \quad j=0,1, \ldots,
$$

and $m$ is a fixed positive real number.

Unlike differential equations, discrete equations with the given initial conditions always have a solution.

In order to establish our main result, we will use the following discrete Gronwall-type inequality.

Theorem 2.1 [9]. Assume that

$$
z(k) \leq C+\sum_{i=0}^{k-1} \sum_{j=1}^{p} a_{j}(i) z\left(i-\sigma_{j}\right)^{m}, \quad k \in Z^{+},
$$

where $m>0,0=\sigma_{1}<\sigma_{2}<\cdots<\sigma_{p}, p \geq 1, C>0, a_{j}(k) \geq 0$ for $j=1,2, \ldots, p$ and $k \in Z^{+}$, and $z(k) \leq C$ for $k=-\sigma_{p},-\sigma_{p}+1, \ldots, 0$.

(a) If $0<m<1$ and $C \leq 1$, then

$$
z(k) \leq C^{m^{k}} \prod_{i=0}^{k-1}\left[1+\sum_{j=1}^{p} a_{j}(i)\right], \quad k \in Z^{+} .
$$

(b) If $m=1$, then

$$
z(k) \leq C \prod_{i=0}^{k-1}\left[1+\sum_{j=1}^{p} a_{j}(i)\right], \quad k \in Z^{+}
$$

(c) If $m>1$, then

$$
z(k) \leq \frac{C}{\left\{1-(m-1) C^{m-1} \cdot \sum_{i=0}^{k-1} \sum_{j=1}^{p} a_{j}(i)\right\}^{1 /(m-1)}}, \quad k \in Z^{+},
$$

provided that

$$
1-(m-1) C^{m-1} \sum_{i=0}^{k-1} \sum_{j=1}^{p} a_{j}(i)>0, \quad k \in Z^{+} .
$$

Assumption 2.2. It is assumed that the unperturbed linear difference equation

$$
u_{j+1}=A_{j} u_{j}, \quad j=0,1, \ldots,
$$

has a bounded growth, that is, there exist real constants $\gamma \geq 1$ and $\alpha>0$ such that

$$
\|\Phi(j, i)\| \leq \gamma \alpha^{j-i}, \quad \forall j \geq i \geq 0,
$$

where $\Phi(k, l)=\prod_{j=l}^{k-1} A_{j}, k>l$, is the fundamental matrix solution of (2.9). 
3. Main results. Now, we are in a position to establish our main results pertaining to the bounded growth and the zero convergence properties of the solutions of (2.1) subject to the conditions (2.2).

THEOREM 3.1. Assume that conditions (2.3) and (2.10) hold. In addition, assume that

$$
\sum_{i=0}^{\infty} \sum_{l=1}^{p} \alpha^{(m-1) i-m \sigma_{l}} q_{l}(i)<\infty
$$

Then,

(a) if $0<m \leq 1$ and $C=\gamma\left\|\tau_{0}\right\| \leq 1$, every solution $u_{j}$ of (2.1), (2.2), such that $\left\|u_{j}\right\| \leq$ $C \alpha^{j}$ for $j=-\sigma_{p},-\sigma_{p}+1, \ldots, 0$, satisfies

$$
\left\|u_{j}\right\| \leq \alpha^{j}\left(\gamma\left\|\tau_{0}\right\|\right)^{m^{j}} \exp \left(\gamma \sum_{i=0}^{\infty} \sum_{l=1}^{p} \beta_{l}(i)\right), \quad j=1,2, \ldots,
$$

where $\beta_{l}(i)=\alpha^{(m-1) i-m \sigma_{l}-1} q_{l}(i)$,

(b) if $m>1$ and

$$
\left\|\tau_{0}\right\| \leq\left\{\frac{\eta}{(m-1) \lambda \gamma^{m}}\right\}^{1 /(m-1)}, \quad \lambda=\sum_{i=0}^{\infty} \sum_{l=1}^{p} \beta_{l}(i),
$$

for some $\eta \in(0,1)$ and $C=\gamma\left\|\tau_{0}\right\|$, every solution $u_{j}$ of (2.1), (2.2) such that $\left\|u_{j}\right\| \leq C \alpha^{j}$ for $j=-\sigma_{p},-\sigma_{p}+1, \ldots, 0$, satisfies

$$
\left\|u_{j}\right\| \leq \frac{\gamma \alpha^{j}}{(1-\eta)^{1 /(m-1)}}\left\|\tau_{0}\right\|, \quad j=0,1,2, \ldots .
$$

Proof. By inductive arguments, we can prove that the unique solution $\left\{u_{j}\right\}_{j=-\sigma_{p}}^{\infty}$ of (2.1), subject to given initial values $u_{0}=\tau_{0}, u_{-1}, \ldots, u_{-\sigma_{p}}$, satisfies

$$
u_{j}=\Phi(j, 0) \tau_{0}+\sum_{i=0}^{j-1} \Phi(j, i+1) f_{i}\left(u_{\left(i-\sigma_{1}\right)}, \ldots, u_{\left(i-\sigma_{p}\right)}\right), \quad j \in Z^{+} .
$$

Hence, by conditions (2.3) and (2.10),

$$
\left\|u_{j}\right\| \leq \gamma \alpha^{j}\left\|\tau_{0}\right\|+\gamma \sum_{i=0}^{j-1} \sum_{l=1}^{p} \alpha^{j-i-1} q_{l}(i)\left\|u_{i-\sigma_{l}}\right\|^{m} .
$$

This yields

$$
\alpha^{-j}\left\|u_{j}\right\| \leq \gamma\left\|\tau_{0}\right\|+\gamma \sum_{i=0}^{j-1} \sum_{l=1}^{p} \alpha^{-i-1} q_{l}(i)\left\|u_{i-\sigma_{l}}\right\|^{m} .
$$

By setting $z(j)=\alpha^{-j}\left\|u_{j}\right\|$ and $\beta_{l}(i)=\alpha^{(m-1) i-m \sigma_{l}-1} q_{l}(i)$, it follows that

$$
z(j) \leq C+\gamma \sum_{l=1}^{p} \sum_{i=0}^{j-1} \beta_{l}(i) z^{m}\left(i-\sigma_{l}\right),
$$

where $C=\gamma\left\|\tau_{0}\right\|$ and $z(j) \leq C$ for $j=-\sigma_{p},-\sigma_{p}+1, \ldots, 0$. 
CASE 1. If $0<m \leq 1$ and $C \leq 1$, then by Theorem 2.1 (a) it follows that

$$
z(j) \leq C^{m^{j}} \prod_{i=0}^{j-1}\left[1+\gamma \sum_{l=1}^{p} \beta_{l}(i)\right] \leq C^{m^{j}} \exp \left(\gamma \sum_{i=0}^{\infty} \sum_{l=1}^{p} \beta_{l}(i)\right),
$$

and the proof of Case 1 is complete.

CASE 2. If $m>1$, proceeding in a similar way to Case 1 , we arrive at the inequality (3.8). Hence, by Theorem 2.1(b), it follows that

$$
z(j) \leq \frac{C}{\left\{1-(m-1) \gamma C^{m-1} \sum_{i=0}^{j-1} \sum_{l=1}^{p} \beta_{l}(i)\right\}^{1 /(m-1)}},
$$

provided that

$$
1-(m-1) \gamma C^{m-1} \sum_{i=0}^{j-1} \sum_{l=1}^{p} \beta_{l}(i)>0 .
$$

Let $\eta \in(0,1)$ be an arbitrary number. We will prove that the condition (3.11) holds for all $\tau_{0}$ satisfying

$$
\left\|\tau_{0}\right\| \leq\left\{\frac{\eta}{(m-1) \lambda \gamma^{m}}\right\}^{1 /(m-1)}=: R,
$$

where $\lambda=\sum_{i=0}^{\infty} \sum_{l=1}^{p} \beta_{l}(i)<\infty$.

Indeed, for all such a $\tau_{0}$, we have

$$
(m-1) \gamma^{m}\left\|\tau_{0}\right\|^{m-1} \sum_{i=0}^{j-1} \sum_{l=1}^{p} \beta_{l}(i) \leq(m-1) \gamma^{m}\left\|\tau_{0}\right\|^{m-1} \sum_{i=0}^{\infty} \sum_{l=1}^{p} \beta_{l}(i) \leq \eta .
$$

Thus,

$$
1-(m-1) \gamma^{m}\left\|\tau_{0}\right\|^{m-1} \sum_{i=0}^{j-1} \sum_{l=1}^{p} \beta_{l}(i) \geq 1-\eta>0 .
$$

Consequently, for all $\tau_{0}$ such that $\left\|\tau_{0}\right\| \leq R$, we have

$$
\begin{aligned}
\left\|u_{j}\right\| & \leq \frac{C \alpha^{j}\left\|\tau_{0}\right\|}{\left\{1-(m-1) \gamma C^{m-1} \sum_{i=0}^{j-1} \sum_{l=1}^{p} \beta_{l}(i)\right\}^{1 /(m-1)}} \\
& \leq \frac{\gamma \alpha^{j}}{(1-\eta)^{1 /(m-1)}}\left\|\tau_{0}\right\|, \quad j \in Z^{+} .
\end{aligned}
$$

Hence the proof of Case 2 is complete.

REMARK 3.2. We want to point out the explicit dependence of the growth constants of the perturbed equation (2.1) upon the growth constants of the unperturbed equation (2.9) and the estimate for the perturbation $f$. Further, the growth rates obtained here are not necessarily decay rates. 
COROLLARY 3.3. Under the assumptions of Theorem 3.1, with $\alpha$ in the open interval $(0,1)$, we have

(i) if $0<m \leq 1$ and $C=\gamma\left\|\tau_{0}\right\| \leq 1$, every solution $u_{j}$ with sufficiently small initial data tends to zero as $j \rightarrow \infty$,

(ii) if $m>1$ and $\left\|\tau_{0}\right\| \leq R$, then the zero solution $u_{j}$ of (2.1), (2.2) is asymptotically stable.

Indeed, the inequality $\left\|u_{j}\right\| \leq \gamma \alpha^{j} /(1-\eta)^{1 /(m-1)}\left\|\tau_{0}\right\|:=K \alpha^{j}\left\|\tau_{0}\right\|, j \in Z^{+}$, shows that for any $\varepsilon>0$, we can choose a suitable number $0<\delta<\min \{R, \varepsilon / K\}$ and a number $N>0$ such that for all $k>N$ and $\left\|\tau_{0}\right\|<\delta$, we have $\left\|u_{j}\right\|<\varepsilon$.

REMARK 3.4. If $A_{k} \equiv A$ is a constant matrix, whose spectral radius is less than 1 , then the zero solution of (2.9) is uniformly asymptotically stable. However, this result cannot be extended to nonautonomous equations (see [7, Theorem 4.4.1]).

4. Special cases. If the system (2.9),

$$
u_{j+1}=A_{j} u_{j}
$$

has slowly varying coefficients, then the condition (2.10) concerning growth of the solutions can be avoided in the case

$$
\left\|A_{k}-A_{j}\right\| \leq q_{k-j} \quad\left(q_{k}=q_{-k}=\text { const }>0, q_{0}=0 ; j, k=1,2, \ldots\right) .
$$

On the other hand, Corduneanu [3] established that for any constant matrix $A$ there exists a constant $\Gamma \geq 1$, independent of the integers $j=0,1,2, \ldots$ such that

$$
\left\|A^{j}\right\| \leq \Gamma \rho^{j}(A), \quad j=0,1, \ldots,
$$

where $\rho(A)$ is the spectral radius of $A$.

In particular, if $A=\left(a_{i j}\right)$ is a triangular constant matrix, then $\Gamma=1$.

Consider in $C^{n}$ the equation

$$
u_{j+1}=A_{j} u_{j}+g_{j}, \quad j \in Z^{+}
$$

where $A_{j}(j=0,1, \ldots)$ are $n \times n$-complex matrices and $g_{j}, u_{j}$ are vectors in $C^{n}$.

THEOREM 4.1. Under condition (4.2), assume that

$$
\begin{aligned}
& \rho_{0}=\sup _{l=0,1, \ldots}\left(\Gamma_{l} \rho\left(A_{l}\right)\right)<1, \\
& S_{0}(A)=\sum_{k=0}^{\infty} q_{k} \rho_{0}^{k}<1, \\
& S_{1}(A ; g)=\sum_{k=0}^{\infty} \rho_{0}^{k}\left\|g_{k}\right\|<\infty,
\end{aligned}
$$


where $\Gamma_{l}$ and $\rho\left(A_{l}\right)$ have the same meaning as the quantities in (4.3) referring to $A$. Then any solution $\left\{u_{j}\right\}_{j=0}^{\infty}$ of (4.4) satisfies the inequality

$$
\sup _{j=1,2, \ldots}\left\|u_{j}\right\| \leq \frac{\rho_{0}\left\|u_{0}\right\|+S_{1}(A ; g)}{1-S_{0}(A)} .
$$

Proof. Rewrite (4.4) as

$$
u_{j+1}-A_{l} u_{j}=\left(A_{j}-A_{l}\right) u_{j}+g_{j}
$$

with a fixed integer $l$. The variation of parameters formula yields

$$
u_{l+1}=A_{l}^{l+1} u_{0}+\sum_{j=0}^{l} A_{l}^{l-j}\left[\left(A_{j}-A_{l}\right) u_{j}+g_{j}\right] .
$$

It follows from (4.2) and (4.3) that

$$
\begin{aligned}
\left\|u_{l+1}\right\| & \leq \rho_{0}^{l+1}\left\|u_{0}\right\|+\sum_{j=0}^{l}\left\|A_{l}^{l-j}\right\|\left[q_{l-j}\left\|u_{j}\right\|+\left\|g_{j}\right\|\right] \\
& \leq \rho_{0}\left\|u_{0}\right\|+\max _{j=0,1,2, \ldots, l}\left\|u_{j}\right\| \sum_{j=0}^{l}\left\|A_{l}^{j}\right\|\left[q_{j}+\left\|g_{l-j}\right\|\right] \\
& \leq \rho_{0}\left\|u_{0}\right\|+\max _{j=0,1,2, \ldots, l}\left\|u_{j}\right\| \cdot \sum_{j=0}^{\infty} q_{j} \sup _{l=0,1,2, \ldots}\left\|A_{l}^{j}\right\|+\sum_{j=0}^{\infty}\left\|g_{j}\right\| \sup _{l=0,1,2, \ldots}\left\|A_{l}^{j}\right\| .
\end{aligned}
$$

Consequently,

$$
\max _{j=0,1,2, \ldots, l+1}\left\|u_{j}\right\| \leq \rho_{0}\left\|u_{0}\right\|+S_{0}(A) \cdot \max _{j=0,1, \ldots, l+1}\left\|u_{j}\right\|+S_{1}(A ; \mathfrak{g}),
$$

and we infer that

$$
\sup _{j=1,2, \ldots}\left\|u_{j}\right\| \leq \frac{\rho_{0}\left\|u_{0}\right\|+S_{1}(A ; g)}{1-S_{0}(A)}
$$

concluding the proof.

Consider the equation

$$
u_{j+1}=A_{j} u_{j}+f_{j}\left(u_{j}\right), \quad j=0,1,2, \ldots
$$

Assume that there are constants $\nu, \mu \geq 0$ such that

$$
\left\|f_{j}(u)\right\| \leq v\|u\|+\mu, \quad j=0,1,2, \ldots .
$$

Denote

$$
\theta_{0}=\frac{\mu}{1-\rho_{0}}, \quad \rho_{0}=\sup _{l=0,1, \ldots}\left(\Gamma_{l} \rho\left(A_{l}\right)\right)
$$

Now we are in a position to formulate the next result of this paper. 
THEOREM 4.2. Under conditions (4.2) and (4.13), assume that

$$
\rho_{0}<1, \quad \psi(A ; f)=\sum_{j=0}^{\infty}\left(q_{j}+v\right) \rho_{0}^{j}<1 .
$$

Then any solution $\left\{u_{j}\right\}_{j=0}^{\infty}$ of (4.12) satisfies the inequality

$$
\sup _{j=1,2, \ldots}\left\|u_{j}\right\| \leq \frac{\rho_{0}\left\|u_{0}\right\|+\theta_{0}}{1-\psi(A ; f)} .
$$

Proof. It can be proved in a similar way to Theorem 4.1, so we will omit the proof.

ACKNOWLEDGMENTS. I thank the referees of this paper for their careful and insightful critique. This research was supported by Fondecyt Chile under Grant 1.030.460 and by Dirección de Investigación, Universidad de Los Lagos.

\section{REFERENCES}

[1] R. P. Agarwal, Difference Equations and Inequalities, Monographs and Textbooks in Pure and Applied Mathematics, vol. 155, Marcel Dekker, New York, 1992.

[2] R. P. Agarwal and P. J. Y. Wong, Advanced Topics in Difference Equations, Mathematics and Its Applications, vol. 404, Kluwer Academic Publishers, Dordrecht, 1997.

[3] A. Corduneanu, The asymptotic behaviour of the solution of some difference equations, Bul. Inst. Politeh. Iaşi. Secț. I. Mat. Mec. Teor. Fiz. 39 (1993), no. 1-4, 45-52.

[4] M. R. Crisci, V. B. Kolmanovskii, E. Russo, and A. Vecchio, Stability of difference Volterra equations: direct Liapunov method and numerical procedure, Comput. Math. Appl. 36 (1998), no. 10-12, 77-97.

[5] S. Elaydi and S. Zhang, Stability and periodicity of difference equations with finite delay, Funkcial. Ekvac. 37 (1994), no. 3, 401-413.

[6] M. I. Gil' and S. S. Cheng, Stability of a time discrete perturbed dynamical system with delay, Discrete Dyn. Nat. Soc. 3 (1999), 56-63.

[7] V. Lakshmikantham and D. Trigiante, Theory of Difference Equations, Mathematics in Science and Engineering, vol. 181, Academic Press, Massachusetts, 1988.

[8] R. Medina, Estimates for the norms of solutions of delay difference systems, Int. J. Math. Math. Sci. 30 (2002), no. 11, 697-703.

[9] V. N. Phat and J. Y. Park, On the Gronwall inequality and asymptotic stability of nonlinear discrete systems with multiple delays, Dynam. Systems Appl. 9 (2000), no. 2, 309321.

[10] M. Pituk, Convergence and uniform stability in a nonlinear delay difference system, Math. Comput. Modelling 22 (1995), no. 2, 51-57.

[11] S. Zhang, Estimate of total stability of delay difference systems, Comput. Math. Appl. 37 (1999), no. 9, 31-38.

[12] S. Zhang and M. Chen, A new Razumikhin theorem for delay difference equations, Comput. Math. Appl. 36 (1998), no. 10-12, 405-412.

Rigoberto Medina: Departamento de Ciencias Exactas, Universidad de Los Lagos, Casilla 933, Chile

E-mail address: rmedina@u1agos.c1 


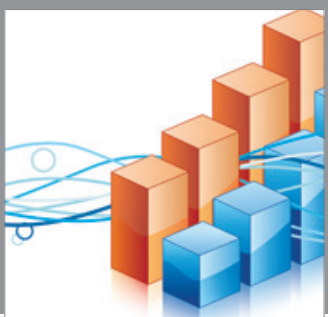

Advances in

Operations Research

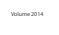

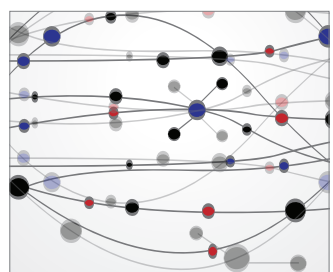

\section{The Scientific} World Journal
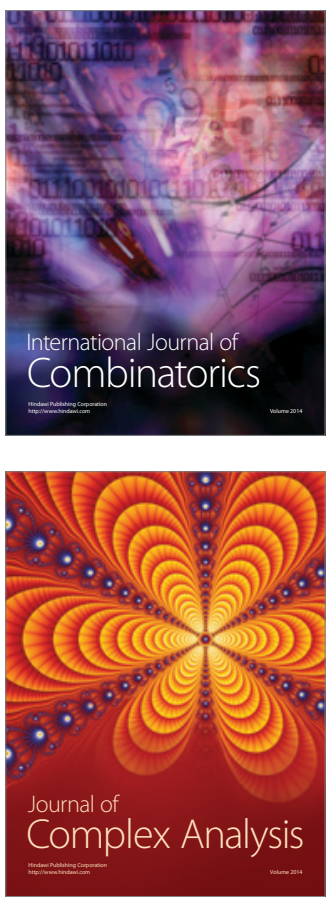

International Journal of

Mathematics and

Mathematical

Sciences
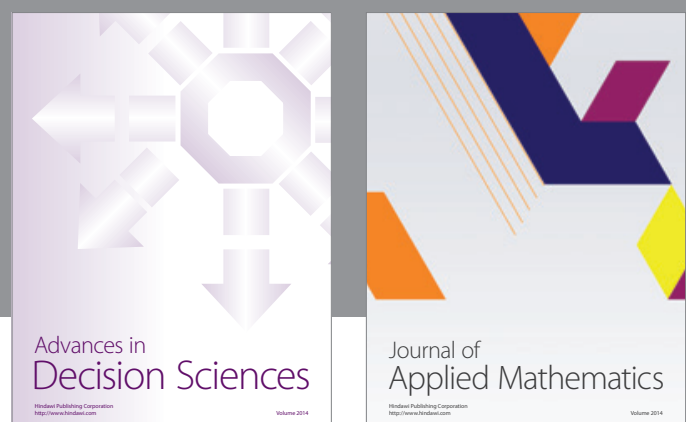

Journal of

Applied Mathematics
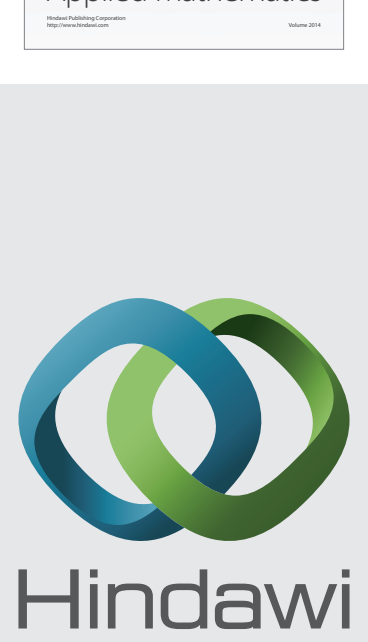

Submit your manuscripts at http://www.hindawi.com
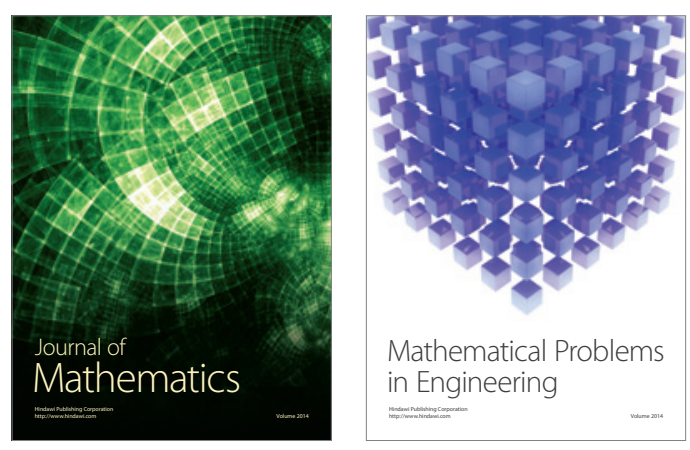

Mathematical Problems in Engineering
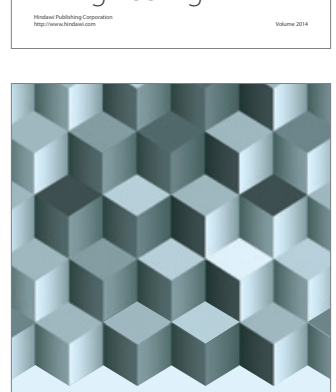

Journal of

Function Spaces
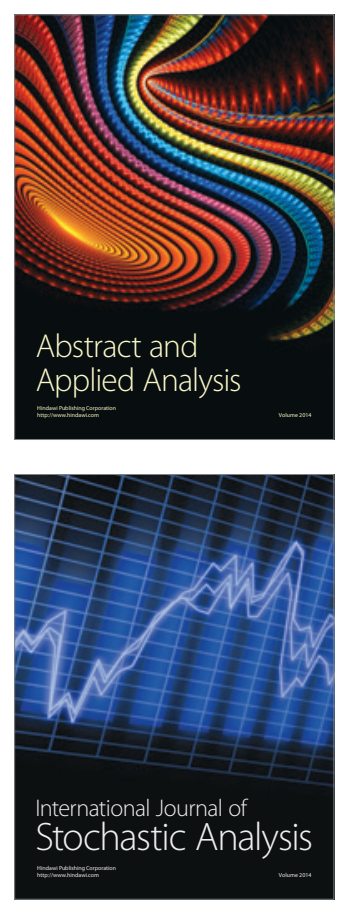

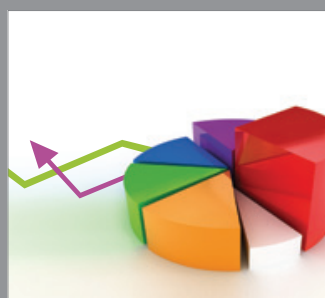

ournal of

Probability and Statistics

Promensencen
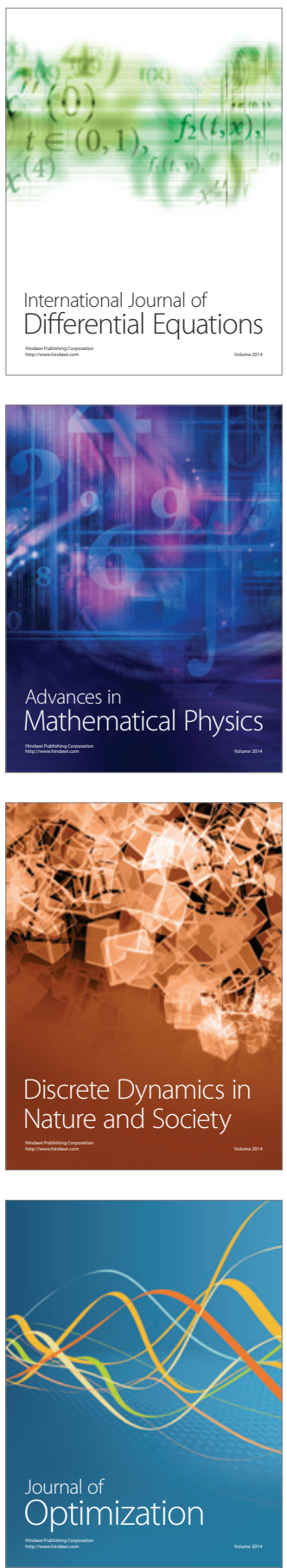